\title{
Mathematik in STRUCTURES
}

\author{
Ralf S. Klessen, Manfred Salmhofer und Anna Wienhard
}

\begin{abstract}
Der Exzellenzcluster STRUCTURES ist ein sehr interdisziplinäres Projekt, das Wissenschaftler aus verschiedenen Bereichen - von der reinen Mathematik bis hin zur Experimentalphysik - zusammenbringt. In diesem Artikel wollen wir die Rolle der Mathematik etwas genauer beschreiben und eines der mathematischen Projekte vorstellen.
\end{abstract}

Der Exzellenzcluster STRUCTURES ist ein interdisziplinäres Projekt, in dem die Mathematik eine Schlüsselrolle spielt. Das Forschungsprogramm von STRUCTURES ist in sieben Comprehensive Projects (CP) organisiert. Jedes dieser Projekte ist selbst schon interdisziplinär und verbindet Wissenschafter unterschiedlicher Bereiche (siehe Abbildung 1 auf S. 179). Die CPs sind aus den vorhandenen methodischen Stärken in Heidelberg erwachsen, wie es der STRUCTURES-Methodenbaum zeigt (Abbildung 1). Wenn man den Methodenbaum genauer untersucht, stellt man fest, dass sowohl Geometrie und Topologie als auch Maschinelles Lernen eine Sonderrolle spielen, da sie in jedes einzelne CP hinein wirken. Es ist in der Tat ein Ziel des Clusters, diese Verbindungen zu vertiefen und moderne Werkzeuge aus der Geometrie und Topologie, als auch des maschinellen Lernens zur Anwendung zu bringen. Dies ist eine Richtung der engen Zusammenarbeit zwischen Mathematik und Physik. Gleichzeitig werden auch Anwendungen in die andere Richtung - von der Physik zur Mathematik untersucht. Die sehr fruchtbare Verbindung von Mathematik und Physik ist eine lange Erfolgsgeschichte, die in unserer Zeit eine Renaissance erlebt hat, in der Ideen aus der Physik zu vollkommen neuen Einsichten in der Mathematik geführt haben. Hier geben wir eine kurze Einführung in eines der mathematischen Forschungsthemen in STRUCTURES, in dem ein interessantes Zusammenspiel zwischen Mathematik und Physik auftritt.

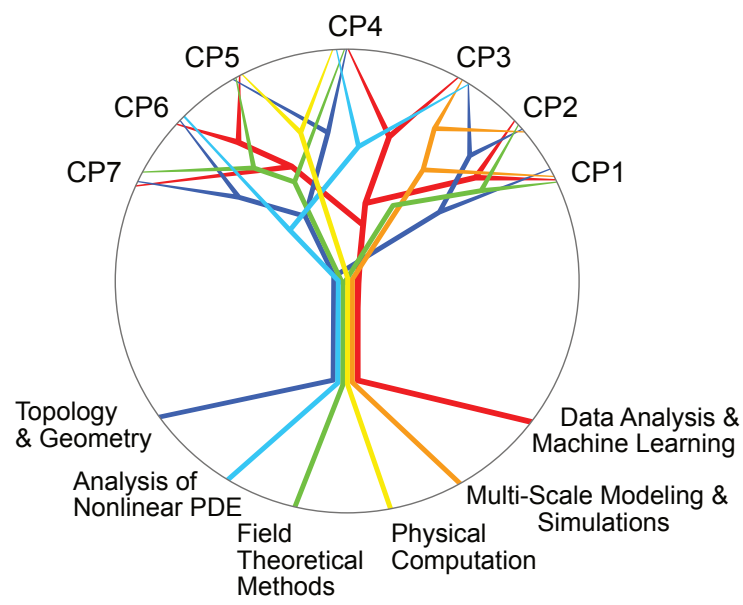

Abbildung 1. Der STRUCTURES-Methodenbaum zeigt, wie die sieben Comprehensive Projects aus den methodischen Stärken in Heidelberg erwachsen sind.
Lokale Systeme

Es gibt eine klassische Korrespondenz zwischen linearen Darstellungen der Fundamentalgruppe einer Mannigfaltigkeit und lokalen Systemen, das sind Verktorbündel mit einem flachen Zusammenhang, auf dieser Mannigfaltigkeit. Für denjenigen, der nicht mit Vektorbündeln und Zusammenhängen vertraut ist, mag dies sehr abstrakt erscheinen, aber lokale Systeme können sehr anschauliche geometrische Objekte beschreiben.

Jede kompakte Fläche ohne Rand trägt eine natürliche (uniformisierende) Geometrie. Diese uniformisierende Geometrie ist eine sphärische, Euklidische oder hyperbolische Riemannsche Metrik. Flächen mit zwei oder mehr Löchern wie der Doppeltorus oder die Oberfläche einer Brezel tragen eine hyperbolische Metrik. Eine solche hyperbolische Metrik auf der Fläche wird im wesentlichen durch ein lokales Systems beschrieben. In einer hyperbolischen Fläche sieht jede Umgebung eines Punktes genau so aus wie eine Umgebung in der hyperbolischen Ebene. Dies ist schwer zu visualisieren, denn würde man eine solche Umgebung in den dreidimensionalen Euklidischen Raum einbetten, sähe es an jedem Punkt so aus, als lebe man auf einem Sattel oder einem Pringle Chip. Eine hyperbolische Fläche lässt sich einfacher visualisieren, wenn man zur universellen Überlagerung, der hyperbolischen Ebene übergeht. Die hyperbolische Ebene lässt sich in vielen verschiedenen Modellen darstellen, z. B. im Poincaré Kreisscheiben-Modell. So wie man einen Torus aus einem Euklidischen Rechteck zusammenkleben kann, kann man eine hyperbolische Fläche mit $g$ Löchern aus einem $4 g-g o n$ in der hyperbolischen Ebene zusammenkleben. Auf diese Weise wird eine hyperbolische Fläche durch eine Pflasterung der hyperbolischen Ebene definiert.

Bekannte Bilder solcher Pflasterungen sind Eschers Kreislimiten (vgl. Abbildung 2). In diesem Spezialfall können wir uns also ein lokales System von Rang 2 auf einer Fläche als eine Pflasterung der hyperbolischen Ebene vorstellen.

Gegeben eine Fläche gibt es nicht nur ein lokales System, das eine hyperbolische Metrik beschreibt. Es gibt unendlich viele hyperbolische Metriken, die alle zusammen einen $6 g$-6-dimensionalen Modulraum bilden, den sogenannten Teichmüller-Raum der Fläche. Der TeichmüllerRaum ist ein facettenreiches und wohl studiertes mathematisches Objekt, das in vielen Bereichen der Mathematik 


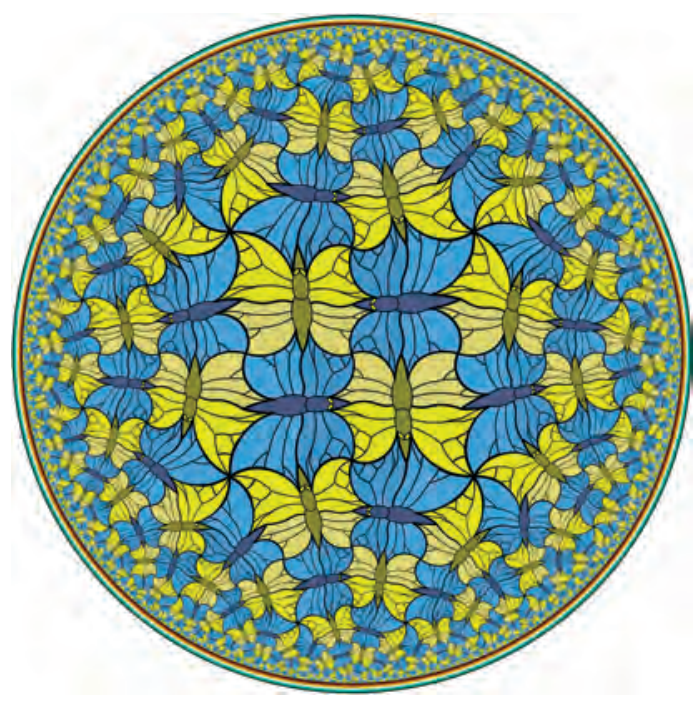

Abbildung 2. Eine Pflasterung der hyperbolischen Ebene durch zweifarbige Schmetterlinge, die in bezüglich der hyperbolischen Geometrie alle gleich sind. (Copyright: Jos Leys)

(und teilweise der theoretischen Physik) eine wichtige Rolle spielt. Durch Wahl einer kombinatorischen Struktur auf der Fläche, zum Beispiel einer Zerlegung der Fläche in Hosenstücke oder in ideale Dreieicke, lassen sich Parametrisierungen des Teichmüller-Raums geben. Einige der Methoden, die hierbei benutzt werden, haben Anwendungen in der Biologie gefunden, insbesondere bei der Untersuchung der Faltungseigenschaften von DNA und Proteinen.

\section{Spektrale Netze und höhere Teichmüller-Theorie}

In den letzten zwanzig Jahren wurde gezeigt, dass es noch viele andere lokale Systeme auf Flächen gibt, die ähnlich der beschriebenen lokalen Systeme im TeichmüllerRaum geometrische Objekte beschreiben. Dies hat zur Entstehung eines neuen, sehr aktiven Gebiets, der höheren Teichmüller-Theorie geführt. Die Modulräume der lokalen Systeme von höherem Rang (größer als zwei), die ähnliche Eigenschaften haben wie die lokalen Systeme, die hyperbolische Metriken beschreiben, heißen höhere Teichmüller-Räume. Es gibt verschiedene Familien solcher höheren Teichmüller Räume: Hitchin-Darstellungen, maximale Darstellungen und allgemeiner positive Darstellungen.

Vor etwa zehn Jahren haben drei Physiker, Davide Gaiotto, Gregory Moore and Andrew Neitzke, eine Beziehung zwischen Hitchin-Darstellungen und speziellen supersymmetrischen Quantenfeldtheorien gefunden. Eine wichtige Rolle in dieser Korrespondenz spielen sogenannte spektrale Netzwerke. Spektrale Netzwerke sind kombinatorisch-dynamische Objekte auf der Fläche, die durch holomorphe Differentiale bestimmt sind. Ein spektrales Netzwerk erlaubt es, sowohl sogenannte BPS-Zustände in den supersymmetrischen Quantenfeld- theorien zu zählen, als auch höhere Teichmüller-Räume zu parametrisieren. Dies gibt eine neue Korrespondenz zwischen $N=2$ vierdimensionalen supersymmetrischen Quantenfeldtheorien der Klasse $S$ auf Seite der Physik und dem Raum der Hitchin-Darstellungen oder positiven Darstellungen auf der mathematischen Seite. Bisher ist jedoch erst ein Teil dieser Korrespondenz verstanden.

Für den Raum der maximalen Darstellungen, der auf mathematischer Seite sehr ähnlich zum Raum der Hitchin-Darstellungen ist, gibt es bisher keine zugehörige physikalische Theorie. Es ist das Ziel eines der mathematischen Projekte in STRUCTURES, die Theorie der maximalen Darstellungen weiter zu entwickeln und die (postulierte) zugehörige physikalische Theorie zu verstehen bzw zu finden. Parametrisierungen des Raums der maximalen Darstellungen legen nahe, dass eine Theorie vektor-wertiger Spektraler Netzwerke hier eine wichtige Rolle spielen wird. Eine zweite Richtung, in die Korrespondenz erweitert werden soll, sind exponentielle Netzwerke, die auf physikalischer Seite entwickelt wurden, um BPS-Zustände in Feldtheorien auf dreidimensionalen Calabi-Yau-Mannigfaltigkeiten zu zählen. Das potentielle mathematische Gegenstück ist hier noch nicht verstanden. Der Raum der lokalen Systeme auf einer Fläche trägt die Struktur einer symplektischen Mannigfaltigkeit. Diese symplektische Struktur, dynamische Systeme und Kompaktifizierungen der höheren Teichmüller-Räume sind ebenfalls mathematische Forschungsthemen innerhalb von STRUCTURES.

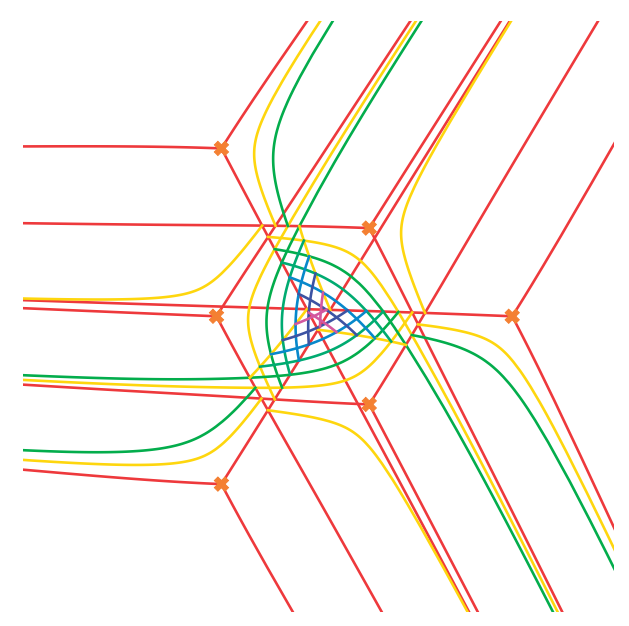

Abbildung 3. Ein spektrales Netz. Spektrale Netze sind kombinatorisch-dynamische Objekte auf der Fläche, mit Hilfe derer man einerseits BPS-Zustände zählen und andererseits höhere Teichmüller-Räume parametrisieren kann. (Copyright: Andrew Neitzke)

\section{Anwendungen}

Die rein mathematischen Forschungsfragen, die innerhalb von STRUCTURES angegangen werden, sind sehr abstrakt und werden wohl erst in der ferneren Zukunft Anwendung finden. Die enge Interaktion zwischen Ma- 
thematik, Physik, Informatik und Astrophysik ist jedoch bereits heute fruchtbar und kann auch die Zeit, in der theoretische mathematische Forschungsergebnisse Anwendungen finden, verkürzen. In STRUCTURES erkunden wir in verschiedenen Projekten neue Anwendungen. Wir nutzen geometrisch-topologische Methoden, wie zum Beispiel persistente Homologie, um Struktur in Daten zu finden und stellen die Frage, inwieweit singuläre Räume hier einen wichtigen Beitrag leisten können. Wir gehen neue Wege im Zusammenspiel zwischen diskreten und kontinuierlichen Strukturen in der (numerischen) Modellierung biophysikalischer Prozesse. Wir nutzen hyperbolische Geometrie und allgemeinere symmetrische Räume im maschinellen Lernen. Wir sind überzeugt, dass die Zusammenarbeit (reiner) Mathematiker mit Wissenschaftlern aus anderen Gebieten ein großes Potential birgt, um Strukturen sowohl in der physischen Welt als auch in Daten besser zu verstehen. STRUCTURES ist ein Ort, an dem diese Zusammenarbeit stattfindet.

\author{
Prof. Dr. Ralf S. Klessen \\ Universität Heidelberg, \\ Zentrum für Astronomie, \\ Institut für Theoretische Astrophysik, \\ Albert-Ueberle-Straße 2, 69120 Heidelberg \\ klessen@uni-heidelberg.de \\ Prof. Dr. Manfred Salmhofer \\ Universität Heidelberg, \\ Institut für Theoretische Physik, \\ Philosophenweg 16, 69120 Heidelberg \\ m.salmhofer@thphys.uni-heidelberg.de \\ Prof. Dr. Anna Wienhard \\ Universität Heidelberg, \\ Mathematisches Institut, MATHEMATIKON, \\ Im Neuenheimer Feld 205, 69120 Heidelberg \\ wienhard@mathi.uni-heidelberg.de
}

Ralf S. Klessen ist Professor für theoretische Astrophysik an der Universität Heidelberg. Er beschäftigt sich mit Sternentstehung, der Dynamik des interstellaren Mediums und der Entwicklung numerischer Methoden in der Astrophysik. In 2002 erhielt er den Ludwig-Biermann-Preis der Astronomischen Gesellschaft und in 2013 einen ERC Advanced Grant zur Untersuchung der Geburt der ersten Sterne.

Manfred Salmhofer geboren 1964 in Graz. Studium der Physik und Mathematik in Graz, Doktorat am MPI für Physik und Astrophysik in München, Promotion 1991. Nach Postdocaufenthalten in Vancouver, Princeton und Leiden ab 1995 Assistenzprofessor am Departement Mathematik der ETH Zürich. Ab 2001 Professor für

Physik an der Universität Leipzig, seit 2008 an der Universität Heidelberg. Forschungsschwerpunkt mathematische Physik und Physik komplexer Systeme.

Anna Wienhard ist Professorin am Mathematischen Institut der Universität Heidelberg und Gruppenleiterin am Heidelberg Institut für Theoretische Studien. Ihr Forschungsschwerpunkt liegt im Bereich der Geometrie und Topologie. Sie beschäftigt sich insbesondere mit Darstellungsvarietäten und geometrischen Strukturen auf Mannigfaltigkeiten. Anna Wienhard verbrachte ihre Postdoc-Zeit am Institut for Advanced Study und der

University of Chicago und war Assistenzprofessorin an der Princeton University. Sie ist Fellow der American Mathematical Society und erhielt 2014 einen ERC Consolidator Grant. 